\title{
Microstructure of low temperature grown AIN thin films on Si(111)
}

\author{
G. W. Auner ${ }^{\mathrm{a})}$ and F. Jin \\ Electrical and Computer Engineering Department, Wayne State University, Detroit, Michigan 48202 \\ V. M. Naik \\ Department of Natural Sciences, University of Michigan-Dearborn, Dearborn, Michigan 48128 \\ R. Naik \\ Department of Physics and Astronomy, Wayne State University, Detroit, Michigan 48202
}

(Received 20 July 1998; accepted for publication 11 February 1999)

\begin{abstract}
AlN thin films were grown on $\mathrm{HF}$-etched $\mathrm{Si}(111)$ substrates at $400-600^{\circ} \mathrm{C}$ by plasma source molecular beam epitaxy. Reflection high energy electron diffraction and transmission electron microscopy studies show that AlN films grown at $400{ }^{\circ} \mathrm{C}$ form an initial amorphous region at the interface, followed by $c$-axis oriented columnar grains with slightly different tilts and twists. AlN films grown at $600^{\circ} \mathrm{C}$ showed a significantly reduced amorphous region near the interface promoting an epitaxial growth of $\mathrm{AlN}$ with $\mathrm{AlN}[0001] \mid \mathrm{Si}[111]$ and $\mathrm{AlN}[01 \overline{1} 0] \| \operatorname{Si}[11 \overline{2}]$ orientations. However, all the films show numerous defects such as stacking faults, dislocations, and grain boundaries. (C) 1999 American Institute of Physics. [S0021-8979(99)00611-8]
\end{abstract}

\section{INTRODUCTION}

Aluminum nitride (AIN) is an important material with many potential applications, because of its wide band gap $(6.2 \mathrm{eV})$, high dielectric strength, high temperature stability, chemical inertness, high thermal conductivity, and high acoustic velocity. ${ }^{1}$ One of the main focuses of AlN research has been on its thin film piezoelectric properties for surface acoustic wave devices. ${ }^{2,3}$ Further, AlN thin films have also been used as buffer layers for the growth of high quality $\mathrm{GaN}^{4}$ and AlGaN films. ${ }^{5}$ AlN films have been grown on silicon substrates using different growth techniques such as chemical vapor deposition (CVD), ${ }^{6,7}$ reactive sputtering, ${ }^{3,8}$ plasma assisted molecular beam epitaxy, ${ }^{9-11}$ and pulsed laser deposition. ${ }^{12}$ The choice of $\mathrm{Si}$ as the substrate for the growth of AlN films has the advantage of its easy integration into the existing device technology. For many device applications, the AlN films must be single crystalline with a smooth surface. During the growth, the substrate temperatures have to be maintained at $>800^{\circ} \mathrm{C}$ in order to obtain good epitaxial AlN films. However, for developing new devices hybridized with $\mathrm{Si}$ devices, it is important to grow AlN films on $\mathrm{Si}$ substrates at low temperatures to avoid thermal damage to $\mathrm{Si}$ devices. Therefore, an investigation of the microstructure of the low temperature grown AlN films on $\mathrm{Si}$ substrates is important.

A few investigators have grown AlN films at lower substrate temperatures employing various methods. ${ }^{6-12}$ For example, Zhang et $a l .{ }^{7}$ have synthesized highly $c$-axis oriented polycrystalline AlN films on $\mathrm{Si}(111)$ substrates via microwave excited electron cyclotron resonance assisted CVD at substrates temperatures ranging from 300 to $400{ }^{\circ} \mathrm{C}$ from the $\mathrm{AlBr}_{3}-\mathrm{N}_{2}-\mathrm{H}_{2} \mathrm{Ar}$ gas system. Meng, Heremans, and Cheng ${ }^{8}$ have studied the growth of AlN on $\mathrm{Si}(111)$ at substrate temperatures ranging from 500 to $800^{\circ} \mathrm{C}$ by ultrahigh vacuum

\footnotetext{
a)Electronic mail: gauner@ece.wayne.edu
}

(UHV) reactive direct current (dc)-magnetron sputtering under a mixture of $\mathrm{Ar}$ and $\mathrm{N}_{2}$ gases. Their results of transmission electron microscopy (TEM), both plan-view and crosssectional studies demonstrate that AlN can be grown epitaxially on $\mathrm{Si}(111)$, with $\mathrm{AlN}[0001] \| \mathrm{Si}[111]$, and $\operatorname{AlN}[11 \overline{2} 0] \mid \operatorname{Si}[2 \overline{2} 0]$. The AlN/Si epitaxy was explained by a 5:4 coincidence growth rather than a lattice matching condition. The lowest temperature required to achieve epitaxy was determined to be $\sim 600^{\circ} \mathrm{C}$, although a considerable in-plane alignment existed in the polycrystalline films grown at $500^{\circ} \mathrm{C}$. At higher growth temperatures, perhaps the surface mobility of the adatoms or precursors is increased and epitaxy is promoted. On the other hand, for growth at low temperatures, limited mobility of the adatoms leads to a polycrystalline (mosaic) texture with preferential columnar orientations. ${ }^{9}$ To our knowledge there have been no crosssectional TEM studies reported in literature on low temperature grown AlN films on $\mathrm{Si}(111)$ substrates to reveal the initial stages of growth. In the present article, we report on the cross-sectional TEM studies of highly $c$-axis oriented AlN thin films grown by plasma source molecular beam epitaxy (PSMBE) deposition ${ }^{13,14}$ on $\mathrm{Si}(111)$ substrates at 400 and $600^{\circ} \mathrm{C}$. While the reflection high energy electron diffraction (RHEED) method was used for in situ structural characterization during the growth process, $\mathrm{x}$-ray diffraction (XRD)/rocking curve measurements were used for ex situ characterization to determine the orientation and texture of the films. The surface morphology of the films was examined by atomic force microscopy (AFM). Cross-sectional TEM studies have been performed to examine the AlN/Si interface and its effect on the subsequent microstructure of the AlN film. A correlation is made between the TEM and RHEED/ XRD observations.

\section{EXPERIMENT}

The AlN films were prepared using a PSMBE deposition system. Details of the deposition system are described 
elsewhere. ${ }^{13,14}$ Briefly, the PSMBE system has a new and innovative MBE deposition source-a hollow cathode source which uses radio frequency (rf) power $(300 \mathrm{~W}$ for this system) to generate a nitrogen/argon plasma. This deposition technique uses a magnetically enhanced hollow cathode lined with the target material. An argon/nitrogen plasma is formed within the hollow cathode. The secondary electrons are confined to the source hollow cathode and do not interact with the substrate, which is approximately $25 \mathrm{~cm}$ away. Furthermore, due to the extreme anisotropy of the kinetically ejected atoms perpendicular to the source wall and the hollow cathode tapered geometry, virtually no high-energy atoms or ions are directed to the substrate. Instead the atoms go through multiple collisions and thermalize. Ions are extracted from the source via an impeller or acceleration bias allowing a controlled energy deposition. Mass spectrometry energy analysis of the nonaccelerated ions ejected from the source indicates a Gaussian energy distribution about the approximate $1 \mathrm{eV}$ range. The PSMBE source, in contrast to planar sputtering, allows enough adatom energy to create AlN (or other nitrides) crystals while minimizing damage to the underlying crystal. The base vacuum of the deposition system is approximately $5 \times 10^{-10}$ Torr, the argon and nitrogen flow rates are respectively, 40 and $20 \mathrm{sccm}$. The dynamic pressure during growth is $1 \mathrm{mTorr}$. The dissociated nitrogen ions and the sputtered aluminum ions are accelerated by a dc biasing $(-12 \mathrm{~V}$ for this system) toward the heated substrate, where they form AlN crystalline films.

The $n$-type $\operatorname{Si}(111)$ substrates were cleaned before loading into the chamber by standard Radio Corporation of America method and dipped in 10\% HF for $30 \mathrm{~s}$ to remove the oxides on the surface. Previous studies ${ }^{15,16}$ have shown that this process passivates the $\mathrm{Si}(111)$ surface against oxidation by hydrogen termination of the Si bonds. The surface is known to be inert for several minutes in air and for several hours in UHV at room temperature. The surface passivation is lost if the substrate is heated to above $550^{\circ} \mathrm{C}$ or by electron bombardment due to hydrogen desorption. ${ }^{17}$ Our previous studies on H-terminated $\mathrm{Si}(111)$ surface showed RHEED patterns with sharp and intense diffraction streaks along with the Kikuchi lines characteristic of a well ordered $1 \times 1$ $\mathrm{Si}(111)$ surface. ${ }^{18}$ We have also observed that the $\mathrm{Si}(111)$ surface remain unreconstructed even after heating up to $850^{\circ} \mathrm{C}$

AlN films of $3000 \AA$ thickness were grown at 400 and $600^{\circ} \mathrm{C}$. The deposition rate was approximately $0.5 \AA / \mathrm{s}$ based on a quartz crystal thickness monitor calibrated using a diamond stylus profilometer. The measured thickness of the film has an error of $\pm 10 \%$. RHEED patterns were continuously monitored during the deposition to gauge the quality and surface structure of the film. Standard XRD $(\theta-2 \theta)$ scans and the rocking curve measurements were performed with a Rigaku powder diffractometer using $\mathrm{Cu} K \alpha$ radiation. A Digital Instruments Nanoscope III Multimode AFM operating in the contact mode was used for AFM studies. TEM cross-section samples were prepared by bonding two films face to face, mechanical thinning, and ion milling. Atomic resolution TEM was performed on a JEOL 4000EX trans-
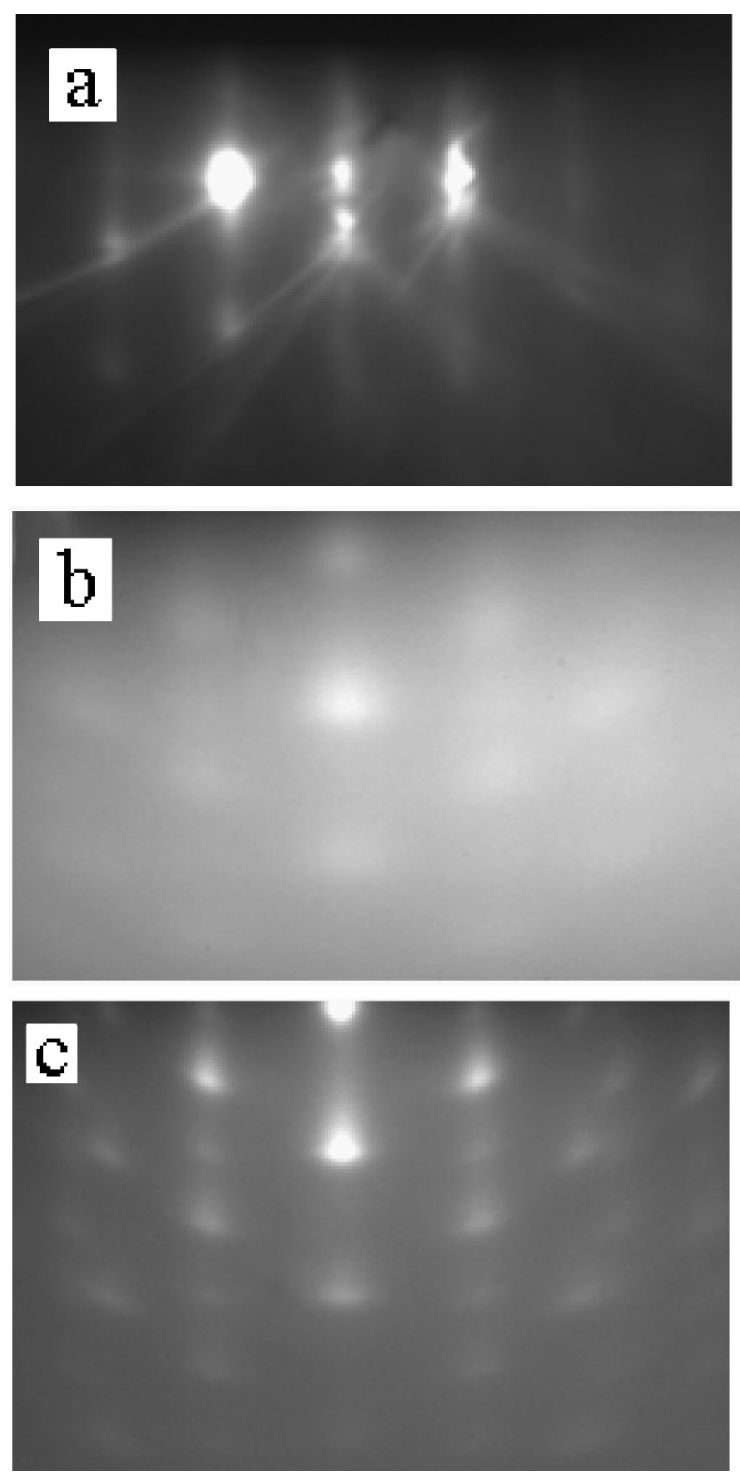

FIG. 1. RHEED patterns viewed along $\mathrm{Si}\langle 112\rangle$ azimuth, during AlN deposition at $400{ }^{\circ} \mathrm{C}$ : (a) $\mathrm{Si}$, (b) after depositing $200 \AA \mathrm{AlN}$, and (c) after depositing $3000 \AA$ 只 AlN.

mission electron microscope, operating at $400 \mathrm{kV}$ enabling a point-to-point resolution better than $1.8 \AA$.

\section{RESULTS AND DISCUSSION}

Figure 1(a) shows the RHEED pattern of a hydrogen terminated $\mathrm{Si}(111)$ surface observed along $\langle 110\rangle$ azimuth of $\mathrm{Si}$ at room temperature. The presence of sharp and intense diffraction streaks along with Kikuchi lines indicates a well ordered $\mathrm{Si}(111)$ surface. AlN was deposited after the substrate was heated to $400^{\circ} \mathrm{C}$. During the initial deposition of AlN film, sharp streaks of $\mathrm{Si}(111)$ were replaced by a bright background, suggesting an amorphous growth. However, after a deposition of $\sim 100$ - $\AA$-thick AlN film, faint diffraction spots appeared. For example, Fig. 1(b) shows the RHEED pattern after a deposition of $200-\AA$-thick AlN film. The pattern became sharper and intense with increasing film thickness. Figure 1(c) shows a snap shot of the RHEED pattern observed along $\mathrm{Si}\langle 110\rangle$ azimuth, when the film thickness was 


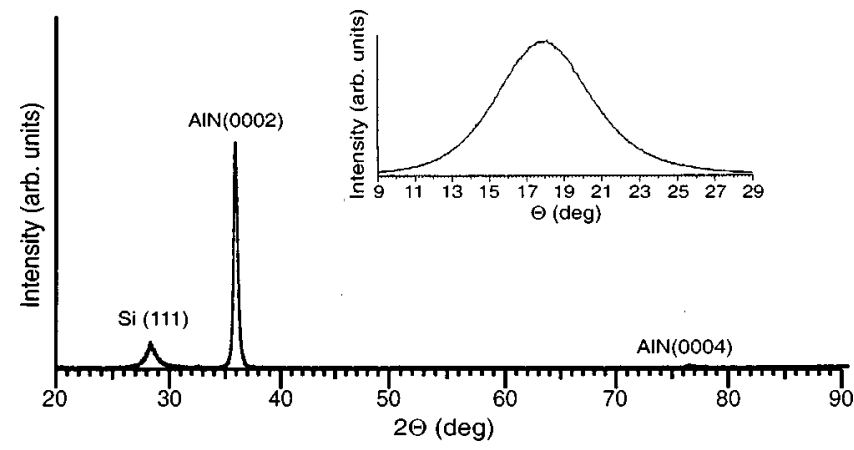

FIG. 2. $\theta-2 \theta$ XRD scan of a 3000 - $\AA$-thick AlN film grown at $400{ }^{\circ} \mathrm{C}$. Inset shows the rocking curve of $\mathrm{AlN}(0002)$ peak.

$3000 \AA$ A. The RHEED pattern was also examined along the $\mathrm{Si}\langle 112\rangle$ azimuth. Surprisingly, the pattern was identical to the one observed along the $\mathrm{Si}\langle 110\rangle$ azimuth. Indeed, the pattern remained essentially the same when viewed along any azimuth of Si. The observed pattern can be indexed (explained later) as a superposition of both $\operatorname{AlN}[2 \overline{1} \overline{1} 0]$ and AlN [01 $\overline{10}]$ zone axes patterns with $c$ axis of AlN oriented along the film normal. The observation of a spotty pattern, which looks the same along any azimuth instead of diffraction rings, indicates that the film is not completely polycrystalline, but rather has a distribution of rotated columnar grains with $c$ axis along the growth direction. In each grain, there is considerable in-plane, alignment similar to the results observed on AlN films grown at $500{ }^{\circ} \mathrm{C}$ by Meng, Heremans, and Cheng. ${ }^{8}$ Their plan-view TEM studies showed a readily perceptible remnant single-crystal selected area diffraction pattern (SADP). The dark-field micrographs taken with remnant $\operatorname{AlN}(10 \overline{1} 0)$ spot showed randomly distributed crystals with a typical grain size of $\sim 100 \AA$. Similar observations were made by Lei et al. ${ }^{19}$ in the case of highly oriented $\mathrm{GaN}$ polycrystalline films grown on $\mathrm{Si}(001)$ by electron cyclotron resonance microwave plasma-assisted MBE.

Figure 2 shows a $\theta-2 \theta$ XRD scan of a 3000 - $\AA$-thick AlN film grown at $400{ }^{\circ} \mathrm{C}$. The XRD pattern shows only AlN(0002) and (0004) peaks. The absence of other AlN reflections indicates a complete texture of the film with AlN[0001]||Si[111] in agreement with the RHEED observations. The full width at half maximum (FWHM) of $\operatorname{AlN}(0002)$ peak is $\sim 0.4^{\circ}$, as compared to $0.15^{\circ}$ observed for $\operatorname{Si}(111)$ reflection. The broadening of $\operatorname{AlN}(0002)$ peak could be due to the presence of micro-stresses and/or to the small crystallite size. The rocking curve was also measured for further investigation of the degree of the $c$-axis orientation. The inset shows the rocking curve scan corresponding to $\operatorname{AlN}(0002)$ peak with a FWHM $\sim 5.7^{\circ}$. This rather large value of FWHM shows a considerable spread in the $c$-axis orientation of AlN columnar grains with respect to the film normal. AFM scans were done in order to see the effect of these grain structures on the film morphology. AFM scans on two different AlN films with thickness of 200 and $3000 \AA$ grown on $\mathrm{Si}(111)$ at $400^{\circ} \mathrm{C}$ are shown in Figs. 3(a) and 3(b), respectively. The 200 - $\AA$-thick AlN film shows a relatively smooth morphology with a root mean square (rms) roughness $\sim 8 \AA$ over an average lateral size of $\sim 300 \AA$. In com-

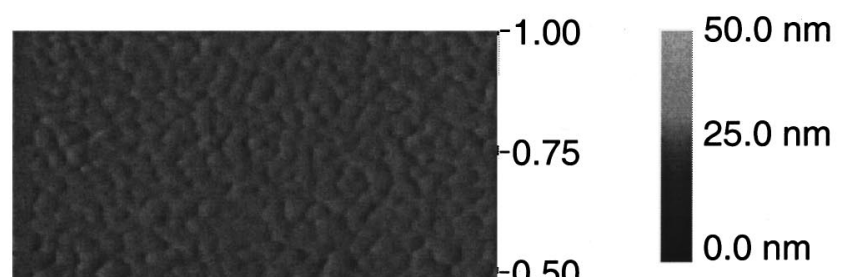

(a)

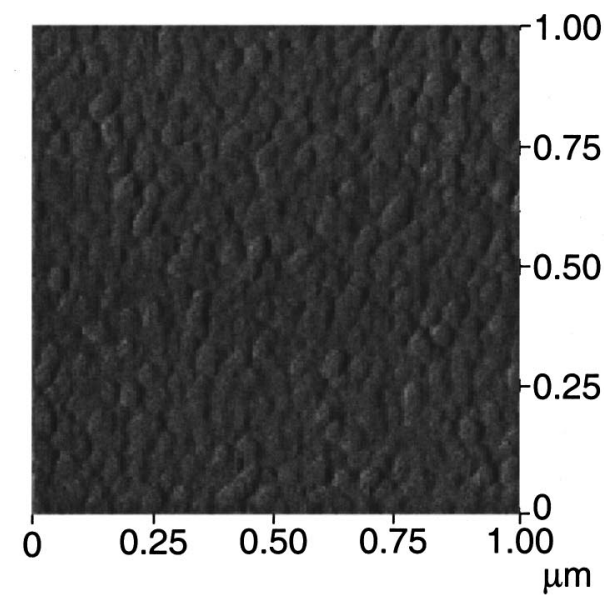

$50.0 \mathrm{~nm}$

$25.0 \mathrm{~nm}$

$0.0 \mathrm{~nm}$

(b)

FIG. 3. AFM scans on two different AlN films with thickness of 200 and $3000 \AA$ grown on $\operatorname{Si}(111)$ at $400^{\circ} \mathrm{C}$.

parison, the 3000- $\AA$-thick film has a rms roughness $\sim 15 \AA$ over an average lateral size of $\sim 400 \AA$. The grain size and roughness seems to increase with the increase in film thickness.

In order to confirm the inferences from RHEED and XRD observations, cross-sectional TEM studies were carried out on the above 3000 - $\AA$-thick AlN film grown on Si(111). These studies reveal how the initial stages of the growth controls the subsequent microstructure of the film. A crosssectional image of the sample near the AlN/Si interface re-

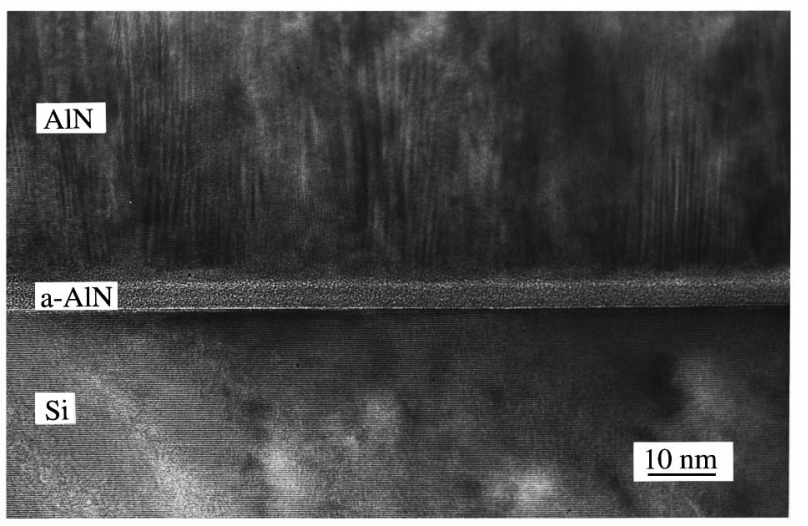

FIG. 4. A cross-sectional image near the AlN/Si interface region of the AlN film grown at $400{ }^{\circ} \mathrm{C}$. The electron beam is parallel to $\mathrm{Si}[11 \overline{2}]$. 
gion, with electron beam parallel to $\mathrm{Si}[112]$, is shown in Fig. 4. The image clearly shows an amorphous region of $\sim 50 \AA$ thickness, followed by growth of crystalline AlN. Kester et $a .^{20}$ have observed a similar initial amorphous region for a $\mathrm{BN}$ film grown on $\mathrm{Si}(100)$ substrate at $400{ }^{\circ} \mathrm{C}$ using electron beam evaporation with simultaneous bombardment of nitrogen and Ar ions. A similar observation has also been made by Yong et al. ${ }^{21}$ during the growth of AlN:H films on $\mathrm{Si}(100)$ substrates at room temperature by rf reactive sputtering. They suggest that the uniformly formed amorphous layer reduces the lattice mismatch and the short range ordering in the amorphous layer provides nucleation sites for the growth of $c$-axis oriented crystallites normal to the substrate. We believe that at lower substrate temperatures, the adatoms do not have enough mobility to align with the Si lattice for epitaxial growth, and hence, form an amorphous layer of AlN, perhaps mixed with $\mathrm{Si}$ in our films. However, we do not expect $\mathrm{SiN}$ formation at these lower temperatures and low ion energies. In fact, the amorphous layer is decreased as the substrate temperature increases indicating a condensed amorphous AlN region rather than a modification of the $\mathrm{Si}$ substrate (higher temperature data described later). Subsequently, crystalline AlN(0002) planes are seen to grow roughly parallel to the substrate surface very similar to the sputtered AlN films grown on glass substrates. ${ }^{22}$ It is believed that the preferential columnar orientation is due to the minimum surface energy of $\mathrm{AlN}(0002)$ relative to other faces. $^{9}$

TEM results are in agreement with our RHEED observations, where only a bright background was seen during the initial stages of AlN growth indicating an amorphous film, followed by diffraction spots corresponding to a crystalline AlN. A careful and closer look at the amorphous region under high resolution TEM revealed that the thickness of the amorphous AlN region is not uniform but varied by $\pm 10 \%$ over a lateral regions of $\sim 200 \AA$. These local variations in thickness seem to create a waviness in the $\operatorname{AlN}(0002)$ planes grown subsequently which leads to a significant tilting and twisting of the $c$-axis oriented grains relative to the film normal. In fact, the vertical fringes observed in the TEM micrograph (Fig. 4) are the rotational Moire patterns ${ }^{23}$ due to the overlapping lattice planes of slightly tilted and twisted grains with respect to the film normal. From the fringe width we estimate that the angle of rotation between the grains to be $\sim 14^{\circ}$ with a grain size of $\sim 200 \AA$. A high resolution TEM micrograph of only AlN region, with electron beam parallel to $\mathrm{Si}[112]$, is shown in Fig. 5. The micrograph reveals numerous defects such as stacking faults, dislocations, and grain boundaries, which are formed when grains containing (0002) AlN planes with slightly different tilts and twists join together.

A SADP taken around the interfacial region of AlN/Si is shown in Fig. 6 along with its indexed pattern. The diffraction pattern shows overlapping $\mathrm{Si}[11 \overline{2}]$ and predominantly $\operatorname{AlN}[2 \overline{1} \overline{1} 0]$ and $\operatorname{AlN}[01 \overline{1} 0]$ zone axis patterns. This indexing of AlN also applies to the RHEED pattern described earlier. In addition, the pattern also reveals the presence of other misaligned regions. Diffraction spots observed along the AlN $\langle 0001\rangle$ direction are significantly intense with their

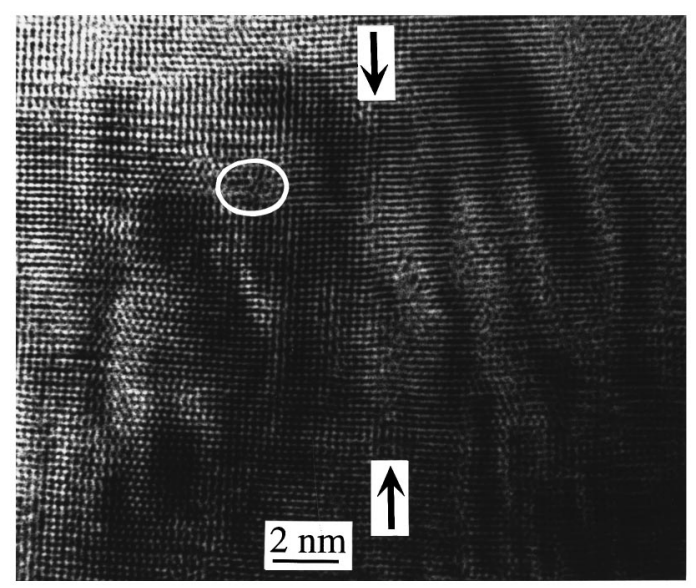

FIG. 5. A high resolution TEM image of only AlN, with electron beam parallel to $\operatorname{Si}[11 \overline{2}]$. The film shows numerous defects, for example, the arrow marks indicate a boundary a region between adjacent the AlN grains with slightly different shift/tilt in the (0002) AlN planes. The circle shows a region of stacking fault.

intensity distributed along an arc of a circle. This implies that the growth is highly $c$-axis oriented with a considerable spread in the $c$-axis orientation with respect to the film normal. This indeed accounts for the observed large FWHM for AlN(0002) XRD rocking curves.

The interpretation that the observed AlN amorphous region is due to low mobility of the adatoms was further tested by performing TEM cross-sectional studies on a $1000-\AA$ thick AlN film grown on hydrogen terminated $\mathrm{Si}(111)$ substrate heated to $600^{\circ} \mathrm{C}$. All other growth conditions were identical to those of AlN films grown at $400{ }^{\circ} \mathrm{C}$. A highresolution cross-sectional TEM image viewed with electron beam parallel to $\mathrm{Si}[1 \overline{1} 0]$ is shown in Fig. 7. The amorphous region in this film is significantly reduced compared to the AlN film grown at $400^{\circ} \mathrm{C}$, allowing an epitaxial growth on $\mathrm{Si}(111)$ surface. Indeed the SADP (inset) shows only AlN[2 $\overline{1} \overline{1} 0]$ spots along with the overlapping $\mathrm{Si}[1 \overline{1} 0]$ zone axis patterns. The epitaxial relationships are $\operatorname{AlN}[0001] \mid \operatorname{Si}[111]$, and $\operatorname{AlN}[01 \overline{1} 0] \| \mathrm{Si}[11 \overline{2}]$ similar to the results of Refs. 8 and 12. The increased temperature seems to promote epitaxial growth. However, a significant waviness and tilting of the $\operatorname{AlN}(0002)$ planes can be still observed in the micrograph, along with the presence of numerous stacking faults, dislocations, and grain boundaries. XRD $\theta-2 \theta$ scan of this film shows only $\operatorname{AlN}(0002)$ and $\operatorname{AlN}(0004)$ peaks very similar to that of Fig. 2, but with a decreased FWHM $\sim 4.4^{\circ}$ compared to $5.7^{\circ}$ for AlN film grown at $400{ }^{\circ} \mathrm{C}$ for $\mathrm{AlN}(0002)$ rocking curve. The film morphology, as determined by AFM, is also very similar to those grown at $400^{\circ} \mathrm{C}$ with a rms roughness of $\sim 10 \AA$ over a lateral size of $400 \AA$.

In summary, we have studied AlN thin films grown on HF-etched $\mathrm{Si}(111)$ substrates by PSMBE deposition at substrate temperatures of $400-600^{\circ} \mathrm{C}$. RHEED and TEM studies show that at $400^{\circ} \mathrm{C}$, AlN film forms an initial amorphous region $(\sim 50 \AA)$ at the interface, followed by $c$-axis oriented columnar grains $(\sim 200 \AA)$ with slightly different tilts and twists. The grain size seems to increase with increase of film 

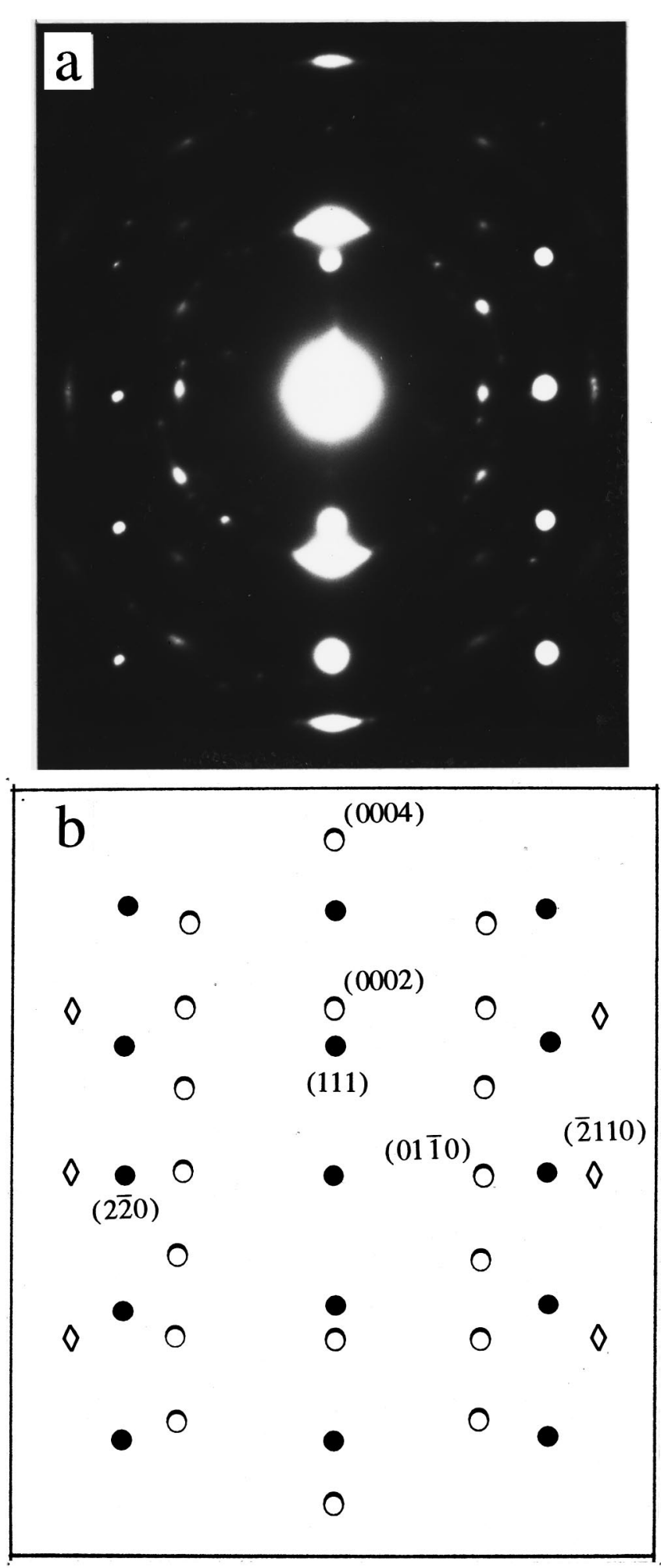

FIG. 6. (a) SADP of overlapping $\mathrm{Si}$ and AlN regions, and (b) indexed pattern of (a). $, \bigcirc, \diamond$ are spots corresponding to $\operatorname{Si}[11 \overline{2}]$, AlN[2 $\overline{1} \overline{1} 0]$, and $\operatorname{AlN}[01 \overline{1} 0]$ zone axis patterns. Remaining spots in (a) are due to misoriented grains and double diffraction.

thickness. AlN film grown at $600{ }^{\circ} \mathrm{C}$ showed a significantly reduced amorphous region near the AlN/Si interface promoting an epitaxial growth of $\mathrm{AlN}[0001] \| \mathrm{Si}[111]$ with $\operatorname{AlN}[01 \overline{1} 0]|| \operatorname{Si}[11 \overline{2}]$. However, all the films show numerous

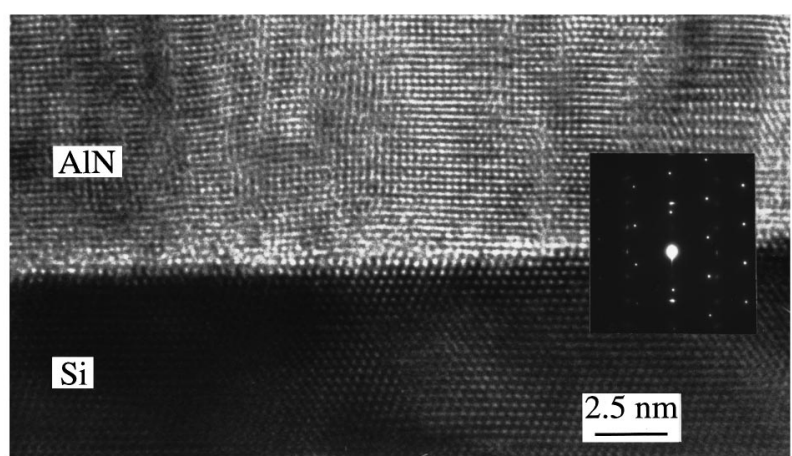

FIG. 7. A high resolution TEM image near the AIN/Si interface region of the AlN film grown at $600^{\circ} \mathrm{C}$, with electron beam parallel to $\mathrm{Si}[01 \overline{1}]$.

defects such as stacking faults, dislocations, and grain boundaries.

\section{ACKNOWLEDGMENTS}

This work was supported by National Science Foundation Grant No. EEC-9420568. The TEM studies were done at the University of Michigan Electron Microbeam Analysis Laboratory.

${ }^{1}$ S. Strite and H. Morkoc, J. Vac. Sci. Technol. B 10, 1237 (1992).

${ }^{2}$ H. Okano, N. Tanaka, K. Shibata, and S. Nakano, Jpn. J. Appl. Phys., Part 1 32, 4052 (1993).

${ }^{3}$ H. Okano, Y. Takahashi, T. Tanaka, K. Shibata, and S. Nakano, Jpn. J. Appl. Phys., Part 1 31, 3446 (1992).

${ }^{4}$ H. Amano, N. Sawaki, I. Akasaki, and Y. Toyoda Appl. Phys. Lett. 48, 353 (1986).

${ }^{5}$ F. A. Ponce, J. S. Major, Jr., W. E. Plano, and D. F. Welch, Appl. Phys. Lett. 65, 2302 (1994).

${ }^{6}$ H. Khan et al., J. Mater. Sci. 39, 4314 (1994).

${ }^{7}$ W. Zhang, Y. Someno, M. Sasaki, and T. Hirai, Jpn. J. Appl. Phys., Part 2 32, L116 (1993).

${ }^{8}$ W. J. Meng, J. Heremans, and Y. T. Cheng, Appl. Phys. Lett. 59, 2097 (1991); Mater. Res. Soc. Symp. Proc. 242, 469 (1992).

${ }^{9}$ K. S. Stevens, A. Ohtani, M. Kinniburgh, and R. Beresford, Appl. Phys. Lett. 65, 321 (1994).

${ }^{10}$ M. Miyauchi, Y. Ishikawa, and N. Shibata, Jpn. J. Appl. Phys., Part 2 31, L1714 (1992).

${ }^{11}$ A. Bourret, A. Barski, J. L. Rouviere, G. Renaud, and A. Barbier, J. Appl. Phys. 83, 2003 (1998).

${ }^{12}$ R. D. Vispute, J. Narayan, H. Wu, and K. Jagannadham, J. Appl. Phys. 77, 4724 (1995).

${ }^{13}$ G. W. Auner, T. D. Lenane, F. Ahmad, R. Naik, P. K. Kuo, and Z. Wu, in Wide Band Gap Electronic Materials (Academic, Netherlands, 1995), p. 329.

${ }^{14}$ P. K. Kuo, G. W. Auner, and Z. L. Wu, Thin Solid Films 253, 223 (1994).

${ }^{15}$ P. Dumas, Y. J. Chabal, and G. S. Higashi, Phys. Rev. Lett. 65, 1124 (1990).

${ }^{16}$ S. S. Iyer, M. Arienzo, and E. de Fresart, Appl. Phys. Lett. 57, 893 (1990).

${ }^{17}$ T. Takahagi, A. Ishitani, H. Kuroda, Y. Nagasawa, H. Ito, and S. Wakao, J. Appl. Phys. 68, 2187 (1990).

${ }^{18}$ R. Naik, C. Kota, B. U. M. Rao, and G. W. Auner, J. Vac. Sci. Technol. A 12, 1832 (1994).

${ }^{19}$ T. Lei, M. Fanciulli, R. J. Molnar, T. D. Moustakas, R. J. Graham, and J. Scanlon, Appl. Phys. Lett. 59, 944 (1991).

${ }^{20}$ D. J. Kester, K. S. Ailey, R. F. Davis, and K. L. More, J. Mater. Res. 8, 1213 (1993)

${ }^{21}$ Y. J. Yong, J. Y. Lee, H. S. Kim, and J. Y. Lee, Appl. Phys. Lett. 71, 1489 (1997).

${ }^{22}$ M. A. Odintzov, N. I. Sushentzov, and T. L. Kudryavtzev, Sens. Actuators A 28, 203 (1991).

${ }^{23}$ H. Hirsch, A. Howie, R. B. Nicholson, D. W. Pashley, and M. J. Whelan, Electron Microscopy of Thin Crystals (Krieger, Malabar, FL, 1977). 